Broman-Fulks, J. J., Berman, M. E., Rabian, B., \& Webster, M. J. (2004). Effects of aerobic exercise on anxiety sensitivity. Behaviour Research and Therapy, 42(2): 125-136. (Feb 2004) Published by Elsevier (ISSN: 1873622X). doi:10.1016/S0005-7967(03)00103-7

\title{
Effects of aerobic exercise on anxiety sensitivity
}

\author{
Joshua J Broman-Fulks, Mitchell E. Berman, Brian A. Rabian, Michael J. Webster
}

\begin{abstract}
Anxiety sensitivity is a known precursor to panic attacks and panic disorder, and involves the misinterpretation of anxiety-related sensations. Aerobic exercise has been shown to reduce generalized anxiety, and may also reduce anxiety sensitivity through exposure to feared physiological sensations. Accordingly, 54 participants with elevated anxiety sensitivity scores completed six 20-min treadmill exercise sessions at either a high-intensity aerobic $(n=29)$ or low-intensity $(n=25)$ level. Self-ratings of anxiety sensitivity, fear of physiological sensations associated with anxiety, and generalized anxiety were obtained at pre-treatment, posttreatment, and one-week follow-up. Results indicated that both high- and low-intensity exercise reduced anxiety sensitivity. However, high-intensity exercise caused more rapid reductions in a global measure of anxiety sensitivity and produced more treatment responders than lowintensity exercise. Only high-intensity exercise reduced fear of anxiety-related bodily sensations. The implications of these findings are discussed.
\end{abstract}




\section{INTRODUCTION}

A growing body of evidence indicates that aerobic exercise is an effective and cost-efficient treatment alternative for a variety of anxiety and mood disorders, including panic disorder (for a review, see Salmon, 2001). However, researchers have not examined whether exercise affects anxiety sensitivity, a known precursor of panic attacks and panic disorder (Ehlers, 1995). Anxiety sensitivity is conceptualized as an enduring fear of anxiety and anxiety-related sensations, brought about from the belief that these sensations can have harmful physical, psychological, or social consequences ( [Reiss and McNally, 1985] and [Taylor, 1999] ). Individuals who are high in anxiety sensitivity have a tendency to misinterpret and catastrophize anxiety-related physiological sensations, which may contribute to or exacerbate the experience of panic ( [Clark, 1986] and [Reiss and McNally, 1985] ). Accordingly, interventions that reduce anxiety sensitivity have the potential to reduce the likelihood that individuals will experience panic attacks or develop panic disorder.

Significant reductions in anxiety sensitivity following ten to twelve sessions of cognitivebehavioral therapy (CBT) have been reported ( [McNally and Lorenz, 1987] and [Telch, Lucas, Schmidt, Hanna, Jaimez and Lucas, 1993] ). CBT treatment packages generally include exposure to feared somatic sensations, arousal reduction, and cognitive-restructuring techniques. Aerobic exercise is believed to reduce anxiety and panic symptoms through similar processes. Specifically, aerobic exercise produces many of the same bodily sensations that often elicit anxiety reactions, such as increases in heart rate, respiration, and perspiration. Repeated exposures to anxiety-related interoceptive stimuli through exercise may therefore extinguish fear responses, accompanied by changes in how these stimuli are interpreted (de Coverley Veale, 1987). Furthermore, aerobic exercise reduces generalized arousal, including resting heart rate and muscle tension (Abadie, 1988). Based on the functional similarities between aerobic exercise and CBT, it is not surprising that some studies have found no differences in the efficacy of CBT and aerobic exercise in the reduction of anxiety ( [Fremont and Craighead, 1987] and [McEntee and Haglin, 1999] ).

In contrast to general anxiety, virtually no attention has been paid to the relation between exercise and anxiety sensitivity. One recent non-experimental study found an inverse relation between exercise frequency and anxiety sensitivity (McWilliams \& Asmundson, 2001). The authors attributed this finding to the notion that exercise produces physiological sensations similar to those feared by individuals with high anxiety sensitivity (e.g., elevated heart rate), and is thus avoided. An alternative interpretation for these findings is that a lack of exposure to these sensations resulting from physical inactivity promotes increased anxiety sensitivity. Regardless, consistent with the literature on general anxiety, it is reasonable to posit that repeated exposure to anxiety-related bodily sensations in the context of aerobic exercise should lead to a reduction in anxiety sensitivity. However, no published experimental study has examined this possibility.

The purpose of the present study was to investigate the effects of aerobic exercise-induced physiological arousal on anxiety sensitivity. Individuals were pre-selected for high anxiety sensitivity, and assigned to six 20-min sessions of high-intensity aerobic exercise (60-90\% of predicted maximal heart rate) or low-intensity walking (one mile per hour). Measures of anxiety 
sensitivity, fear of anxiety-related physiological sensations, and state and trait anxiety were obtained at pre-treatment, post-treatment, and one-week follow-up. Previous research has shown that both high-intensity aerobic and light walking exercise programs reduce generalized anxiety (Sexton, Maere, \& Dahl, 1989). Accordingly, we hypothesized that participants in the high- and low-intensity exercise conditions would both show improvement on all anxiety measures from pre- to post-treatment, and gains would be maintained at follow-up. However, it was expected that the high-intensity exercise group would show significantly more improvement on these measures in comparison with the low-intensity comparison group.

\section{METHOD}

\subsection{Participants}

Participants were 54 students (41 women), ages 18-51 ( $M=21.17, \mathrm{SD}=5.11)$. Volunteers received course credit for participation. Approximately 550 students were screened, of which 59 potential participants met the study entry criteria (see below). Of these, five declined participation, citing scheduling problems or lack of interest. To be included in the study, participants had to: (1) achieve a score of 25 or more (>0.75 standard deviations above the mean) on the Anxiety Sensitivity Index (ASI; Peterson \& Reiss, 1992), (2) be at least 18 years of age, and (3) be in good general health. Exclusion criteria included: (1) a health condition that would preclude aerobic exercise (assessed using the Physical Activity Readiness Questionnaire: PAR-Q; Shepherd, Cox, \& Simper, 1981), (2) current involvement in psychotherapy, (3) current use of any psychotropic medication, and (4) current involvement in an aerobic exercise program. Current exercise involvement was defined as more than one aerobic exercise session per week. The ASI cutoff score for this study was slightly more conservative than cutoff scores used in previous studies (e.g., Maller \& Reiss, 1992). No participant attrition occurred during this study. The consent process and protocol were reviewed by the Institutional Review Board for the Protection of Human Subjects at The University of Southern Mississippi.

\subsection{Instruments}

The ASI (Peterson \& Reiss, 1992) is the most widely used measure of anxiety sensitivity and is believed to assess three first-order factors: (1) fear of physical symptoms of anxiety, (2) fear of cognitions associated with anxiety attacks, and (3) fear of publicly observable symptoms of anxiety, and a general second-order factor (Zinbarg, Barlow, \& Brown, 1997). The psychometric properties of the ASI are well-established and are only briefly mentioned here. The ASI has relatively good stability across two-weeks, with a test-retest correlation of 0.75 , and good internal consistency, with alpha scores ranging between 0.82 and 0.91 . The ASI has also been shown to possess adequate criterion-related validity, and discriminates individuals with anxiety disorders from non-cases (Reiss, Peterson, Gursky, \& McNally, 1986). 
Given that we were particularly interested in reducing fear of interoceptive cues associated with anxiety sensitivity, the Body Sensations Questionnaire (BSQ; Chambless, Caputo, Bright, \& Gallagher, 1984) was included as a primary outcome measure. The BSQ possesses good internal consistency (alpha=0.87), and moderate to good test-retest reliability $(r=0.67)$ over a one-month time period. The BSQ has also been shown to provide independent information above measures of general anxiety, and discriminates individuals with anxiety disorders (Chambless et al., 1984).

Although we did not pre-select individuals based on general anxiety, previous studies have demonstrated that participation in aerobic exercise leads to decreases in state and trait anxiety (e.g., [Fremont and Craighead, 1987] , [McAuley, Mihalko and Bane, 1996] and [McEntee and Haglin, 1999] ). To attempt to replicate these findings in individuals with high-anxiety sensitivity, the State-Trait Anxiety Inventory (STAl; Spielberger, Gorsuch, Lushene, \& Jacobs, 1983) was included in the battery of questionnaires. The psychometric properties of the STAI are welldocumented (e.g., Spielberger et al., 1983).

A brief demographic questionnaire was also completed during the screening sessions to obtain information on participant age, race, gender, exercise, panic history, current use of medications, and history of treatment for anxiety disorders.

\subsection{Procedure}

Potential participants were screened in groups, and completed a screening consent form, the ASI, and the demographic questionnaire. Participants who met the selection criteria were informed by telephone of their acceptance into the study, and of the general expectations for participation. Participants were told that the purpose of the present study would be to examine the effects of different rates of physical activity on general mood states. In accordance with the recommendations of the American College of Sports Medicine (ACSM, 2000), volunteers who met the study entry criteria were administered the PAR-Q to ensure their safety and to determine if medical clearance was necessary (no participant met PAR-Q criteria requiring medical clearance).

Participants were randomly assigned to the high-intensity aerobic exercise condition or the lowintensity walking comparison condition. Participants completed the ASI, BSQ, and STAI (order of presentation of the questionnaires was randomized) over three assessment sessions: immediately before the first exercise session, after a five-minute cool down period following the final exercise session, and at a one-week follow-up session. In addition, the BSQ was administered before exercise sessions 2-5.

Participants wore a Polaris heart monitor during each exercise session to assess heart rate. Each session began with two minutes of stretching exercises and a two-minute treadmill warmup. Those in the high-intensity exercise condition were then asked to briskly walk or jog on a treadmill at a speed that produced exercise heart rates between $60 \%$ and $90 \%$ of the individual's age-adjusted predicted maximal heart rate, as recommended by the ACSM (2000) 
for optimal aerobic exercise conditions. The lower- and upper-bound for each individual's aerobic heart rate range was computed using the following formula: (220-age $) \times(0.60$ [lowerbound] or 0.90 [upper-bound]). Treadmill speed was adjusted as necessary to ensure that participants in the high-intensity exercise group remained within the lower- and upper-bounds of aerobic exercise for the duration of each exercise session. High-intensity exercise participants were encouraged to increase their treadmill speed and heart rates with each subsequent session. A brief cool down period followed treadmill sessions.

Each exercise session lasted $20 \mathrm{~min}$, and was repeated 2-4 times per week for a total of six exercise sessions across two weeks. The majority of participants (87\%) completed three exercise sessions per week. The comparison group completed a similar protocol except treadmill speed was maintained at one-mile per hour so participant heart rate never reached $60 \%$ of maximum. To accommodate participants, yet minimize variability in exercise exposure, sessions were scheduled at the convenience of the participant, but during weekly business hours only.

Research has indicated that significant reductions in anxiety can occur following a single exercise session (Roth, 1989). In contrast, CBT and other therapeutic approaches may require up to 10 sessions for a measurable therapeutic effect. Therefore, to ensure sufficient opportunity for repeated exposure to physiological sensations, yet minimize the number of sessions required, six sessions of exercise exposure were employed. For this initial study, a one-week follow-up session was used to determine if any observed effects continued after the completion of the exercise sequence.

Exercise sessions were conducted in a large laboratory which primarily contained exercise and other health-related equipment. During sessions, only the researcher and the individual participant were present. The researcher's primary function was to administer questionnaires, monitor participant heart rates, control treadmill speed, and offer assistance if necessary. No other activities were allowed during the exercise sessions to minimize the possibility that participants would be distracted from observation of bodily sensations. No further instructions were provided.

\section{RESULTS}

The study hypotheses were tested using separate exercise group by assessment session mixed-model analyses of variance (ANOVAs) for each of the dependent measures. The repeated-measures factor had three levels (pre-intervention, post-intervention, and follow-up) for all analyses except the BSQ, which was also completed before treadmill sessions 2-5 (for a total of seven assessment points). If violations of the sphericity assumption were detected, significance tests were also conducted using the Greenhouse-Geisser correction method. Corrected and uncorrected analyses produced the same pattern of significant and nonsignificant effects. Therefore, to simplify data presentation, uncorrected dfs are reported. Because multiple omnibus ANOVAs were conducted, Bonferroni corrected $p$ values were used $(p=0.01)$ to assess main effects and interaction terms. Significant interactions were analyzed by 
examining within-group simple effects, also corrected for number of analyses performed, followed by post hoc mean comparisons using Tukey's HSD procedure.

\subsection{Demographic characteristics and pre-treatment scores}

Independent t-tests and chi-square tests indicated that the two treatment groups were comparable at pre-treatment on all demographic variables (all ps>0.10; see Table 1). Independent sample t-tests revealed no significant differences between the two treatment groups on any of the outcome measures at pre-treatment (Table 2).

Table 1. Demographic characteristics as a function of high-intensity versus low-intensity exercise conditions

\begin{tabular}{|c|c|c|c|c|c|c|}
\hline \multirow[t]{2}{*}{ Variable } & \multicolumn{3}{|c|}{ High-intensity group $(n=29)$} & \multicolumn{3}{|c|}{ Low-intensity group $(n=25)$} \\
\hline & $n(\%)$ & Mean & SD & $n(\%)$ & Mean & SD \\
\hline Age & & 20.76 & 3.16 & & 21.64 & 6.75 \\
\hline \multicolumn{7}{|l|}{ Gender } \\
\hline Men & $6(21)$ & & & 7 (28) & & \\
\hline Women & $23(79)$ & & & $18(72)$ & & \\
\hline \multicolumn{7}{|l|}{ Race } \\
\hline Caucasian & $16(55)$ & & & $9(36)$ & & \\
\hline African-American & $13(45)$ & & & $3(52)$ & & \\
\hline Asian & - & & & $2(8)$ & & \\
\hline Native American & - & & & $1(4)$ & & \\
\hline \multicolumn{7}{|l|}{ Panic History } \\
\hline Yes & $9(31)$ & & & $9(36)$ & & \\
\hline No & $20(69)$ & & & $16(64)$ & & \\
\hline
\end{tabular}

Note. The groups did not differ on any of the above variables. 
Table 2. Group means and standard deviations at each assessment period for outcome measures

\begin{tabular}{|c|c|c|}
\hline Measure & High-intensity $(n=29)$ & Low-intensity $(n=25)$ \\
\hline \multicolumn{3}{|l|}{ ASI } \\
\hline Pre-treatment & 34.17 (6.30) & $31.44(5.06)$ \\
\hline Post-treatment & $25.03(9.71)$ & 28.56 (6.01) \\
\hline Follow-up & $23.48(9.44)$ & 25.32 (6.32) \\
\hline \multicolumn{3}{|l|}{ BSQ } \\
\hline Pre-treatment & $2.52(0.53)$ & $2.69(0.57)$ \\
\hline Post-treatment ${ }^{\star \star}$ & $1.96(0.53)$ & $2.50(0.65)$ \\
\hline Follow-up** & $2.00(0.52)$ & $2.52(0.60)$ \\
\hline \multicolumn{3}{|l|}{ STAI-State } \\
\hline Pre-treatment & $35.10(9.07)$ & $35.12(9.83)$ \\
\hline Post-treatment ${ }^{\star}$ & 32.03 (8.15) & 38.24 (11.66) \\
\hline Follow-up & 35.72 (11.35) & 38.08 (12.34) \\
\hline \multicolumn{3}{|l|}{ STAI-Trait } \\
\hline Pre-treatment & 41.67 (9.54) & $42.72(10.74)$ \\
\hline Post-treatment & $38.79(7.82)$ & $42.32(10.53)$ \\
\hline Follow-up & $40.14(9.46)$ & 42.28 (11.89) \\
\hline
\end{tabular}

ASI=Anxiety Sensitivity Index; BSQ=Body Sensations Questionnaire; STAI=State-Trait Anxiety Inventory. The BSQ was also administered before exercise sessions 2-5 (see Fig. 1). No pre-treatment group differences emerged for any of the above measures. ${ }^{*} p<0.05$.

${ }^{* *} p<0.01$.

\subsection{Manipulation check: aerobic exercise}

To determine if the participants in the high-intensity exercise group achieved exercise heart rates consistent with the intensity range for aerobic exercise, as recommended by the ACSM (2000), and that those in the comparison condition did not, average heart rates for the two groups were examined. Average heart rate for the high-intensity group $(M=139.38, S D=10.48)$ was within the age-predicted aerobic range of 119-179 beats per minute, whereas the lowintensity group $(M=103.14, S D=9.68)$ did not achieve heart rates within the age-predicted aerobic range of 119-178 beats per minute. An independent samples $t$-test revealed that average group heart rates were significantly different, $t(52)=13.13, p<0.0001$. 


\subsection{Exercise effects on anxiety sensitivity measures}

Pre-treatment, post-treatment, and follow-up means and standard deviations for the outcome measures are presented in Table 2. Recall that participants completed two measures of anxiety sensitivity, the Anxiety Sensitivity Index and the Body Sensations Questionnaire. To determine if significant changes in anxiety sensitivity occurred as a function of aerobic exercise, separate mixed-model ANOVA were performed for these outcome measures.

\subsubsection{Anxiety Sensitivity Index}

A $2 \times 3$ (exercise group $\times$ assessment time) mixed-model ANOVA performed on mean ASI scores revealed a significant main effect for assessment session, $F(2,104)=49.98, p<0.001, \eta^{2}=0.49$, with higher ASI scores found at pre-treatment $(M=32.91, S D=5.87)$ than at post-treatment $(M=26.67, S D=8.33)$ and follow-up $(M=24.33, S D=8.13)$. Post hoc analyses indicated that the decrease from pre- to post-treatment was significant, as was the decrease from pre-treatment to follow-up. However, the decrease from post-treatment to follow-up did not attain significance.

A group by time interaction was also found, $F(2,104)=6.99, p=0.001, \eta^{2}=0.12$. A significant simple effect for assessment session emerged for the high-intensity exercise group, $F(2$, $56)=42.50, p<0.001, \eta^{2}=0.60$. Post hoc tests revealed that ASI scores declined from preintervention to post-intervention. Although the high-intensity group maintained this decrease at follow-up, follow-up ASI scores were not significantly different compared to post-treatment scores. An assessment session effect was also found for the low-intensity comparison group, $F(2,48)=13.72, p<0.001, \eta^{2}=0.36$. Post hoc tests indicated that the comparison group did not experience a significant change in ASI scores from pre- to post-treatment. Follow-up scores were significantly lower compared to post-treatment scores, however.

The clinical significance of high-intensity exercise can be examined by comparing the number of "treatment" responders in the two groups, both at post-intervention and follow-up. Treatment responders were defined as those who had decreases in ASI scores of one standard deviation (9 points) or more at each of these time-points. Too few participants $(n=5)$ decreased $1.96 \mathrm{SD}$ or more (a criterion suggested by Jacobson \& Truax, 1991) to allow meaningful analysis.

However, decreases of one SD or more were not uncommon. It is reasonable to posit that this represents a meaningful change, given the ASI inclusion cut-off score. At post-treatment, 15 of 29 (52\%) participants in the high-intensity group and five of 25 (20\%) participants in the lowintensity comparison group reported a decrease of this magnitude, representing a significant difference in treatment responders, $X^{2}(1, N=54)=5.80, p<0.05$. At follow-up, 18 participants in the high-intensity group (62\%) and seven participants in the comparison group (28\%) reported a one SD or more decrease in ASI scores from pre-treatment. Again, more high-intensity exercise participants responded to treatment than did low-intensity exercise participants, $X^{2}(1$, $N=54)=6.27, p=0.01$. 


\subsubsection{Body Sensations Questionnaire}

The BSQ was administered to participants to assess fear of anxiety-related physiological sensations. In addition to the pre-treatment, post-treatment, and follow-up assessments, the BSQ was also administered to participants before beginning the exercise task in sessions $2-5$, which allowed for a more comprehensive view of any changes that may have taken place. $A$ $2 \times 7$ (exercise group $\times$ assessment time) mixed-model ANOVA revealed a significant main effect for assessment session, $F(6,312)=13.30, p<0.001, \eta^{2}=0.20$, and a main effect for exercise group, $F(1,52)=9.44, p<0.01, \eta^{2}=0.15$. Mean BSQ scores for the high-intensity exercise group $(M=2.12, \mathrm{SD}=0.10)$ were significantly lower on average compared to the low-intensity comparison group $(M=2.56, \mathrm{SD}=0.11)$.

A significant interaction effect between group and exercise session was also found, $F(6$, $312)=4.56, p<0.001, \eta^{2}=0.08$. Fig. 1 illustrates the treatment effects for the two groups over the seven assessment sessions. The simple effect analysis for the exercise group was significant, $F(6,168)=13.35, p<0.001, \eta^{2}=0.32$. Post hoc tests revealed a significant decrease in pretreatment BSQ scores following the initial exercise session, with scores remaining significantly lower compared to pre-treatment levels through each of the following sessions. In contrast, the simple effect analysis for the low-intensity comparison condition was not significant.

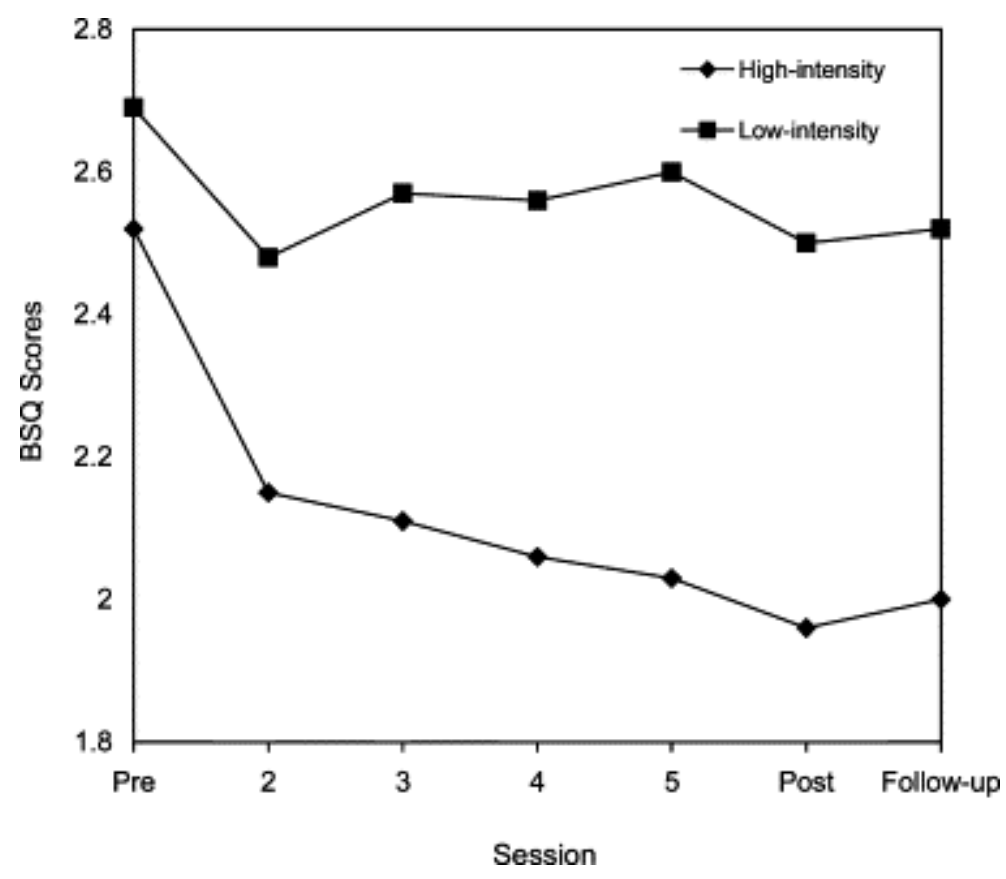

Fig. 1.

Mean BSQ scores for high- and low-intensity exercise groups at pre-intervention, before each exercise session, post-intervention, and at a one-week follow-up session. 


\subsection{State and trait anxiety}

Separate $2 \times 3$ mixed-model ANOVAs were performed on state and trait anxiety scores. No significant effects emerged from these analyses.

\subsection{Associations among anxiety measures}

Pearson-product moment correlations among the pre-intervention anxiety measures were calculated. No significant correlations were found between baseline ASI scores and either STAIS or STAI-T scores. In contrast, baseline BSQ scores were related to the ASI $(r=0.33, p<0.05)$, STAI-S $(r=0.31, p<0.05)$, and STAI-T $(r=0.32, p<0.05)$. A strong correlation was found between the STAI subscales $(r=0.80, p<0.01)$, which is consistent with previous reports (e.g., Spielberger et al., 1983). To determine if baseline BSQ scores uniquely predict ASI scores controlling for state and trait anxiety, we conducted a hierarchical multiple regression, entering the STAI subscales simultaneously in the first block, followed by the BSQ in the second block. Block one was non-significant, $R^{2}=0.05, F(2,51)=1.26, p=0.29$. $\Delta R^{2}$ for block two was significant, $F(1$, $50)=4.42, p<0.05$. Thus, the BSQ predicts ASI scores controlling for general anxiety.

\section{DISCUSSION}

The purpose of the present study was to investigate the effects of aerobic exercise on anxiety sensitivity. We hypothesized that participants in the high-intensity exercise condition and the low-intensity comparison condition would both show improvement in anxiety sensitivity, but that the high-intensity exercise group would show more improvement at post-treatment and followup compared to the comparison group. Results indicated that high-intensity exercise and lowintensity exercise were both successful, on average, at decreasing overall anxiety sensitivity at follow-up as reported on the ASI. High-intensity exercise, however, had several distinct advantages over low-intensity exercise. First, high-intensity exercise produced more rapid reductions in ASI scores. Second, high-intensity exercise yielded more than twice as many "treatment" responders.

With respect to BSQ scores, only high-intensity exercise reduced fear of anxiety-related physiological sensations. Specifically, the high-intensity exercise group reported significantly less fear of anxiety-related bodily sensations at post-intervention compared to their counterparts in the low-intensity comparison group, and these group differences were maintained at followup. Interestingly, these differences emerged by the beginning of the second exercise session. Thus, high-intensity exercise may be especially effective in the rapid reduction of fear of physiological arousal in high-anxiety sensitivity individuals.

The finding that two weeks of low-intensity exercise reduced global anxiety sensitivity, as assessed by the ASI, is worthy of discussion. Although participants in the low-intensity condition did not achieve heart rates in the aerobic range, it appears that even this low level of physical activity can reduce anxiety sensitivity for some individuals. This finding is similar to that reported 
by Sexton et al. (1989), who found comparable reductions in general anxiety for participants who exercised by jogging and those who participated in light walking. The authors speculated that some minimum amount of physical activity might be biologically necessary to keep anxiety at healthy levels. The present results indicate that this may also be true for anxiety sensitivity. It may also be the case that more robust aerobic exercise-induced reductions in global anxiety sensitivity may be achieved with the addition of other treatment components (e.g., cognitive restructuring).

Previous studies have reported that participation in an aerobic exercise program can reduce state and trait anxiety (e.g., [Fremont and Craighead, 1987], [McEntee and Haglin, 1999] and [McAuley, Mihalko and Bane, 1996] ). However, we were unable to replicate these findings. One potential explanation for this is that our participants were not pre-selected based on high general anxiety scores. Although it may be expected that individuals high in anxiety sensitivity would also, on average, have elevated state and trait anxiety scores, this was not the case in the present study. Our STAI scores were close to the mean scores found in samples with similar characteristics (e.g., Spielberger et al., 1983). In addition, no significant associations between ASI scores and either the STAI-S or STAI-T were found. Thus, further reductions in state and trait anxiety may not have been possible. In contrast to STAI scores, pre-selection on the ASI did produce elevated mean BSQ scores. This finding, coupled with the results of a hierarchical multiple regression analysis showing that BSQ scores uniquely predict ASI scores, supports the notion that the ASI and BSQ are more closely related compared to measures of general anxiety.

When participants are selected based on extreme scores, regression to the mean is a potential threat to the internal validity of a study. An active control condition was used to limit threats to study construct validity. When using this control strategy, beneficial changes in the experimental condition must exceed those in the comparison condition to rule out statistical regression. In the present study, high-intensity exercise produced significant improvement in general anxiety sensitivity scores as measured by the ASI at post-intervention, whereas low-intensity exercise did not. However, both groups were equivalent at follow-up, which may reflect either statistical regression or a delayed effect of low-intensity exercise. Reductions in BSQ scores for the highintensity exercise group at post-intervention and follow-up, coupled with stable scores for the control group and the well-documented psychometric properties of the ASI (i.e. relatively high two-week test-retest reliability of 0.75; Peterson \& Reiss, 1992) provide compelling evidence that the effects of high-intensity exercise were not due to statistical regression, at least for fear of physiological sensations. We recommend future researchers include an assessment-only control condition, in addition to an active control group, to address these issues in more depth.

Due to the promising nature of the present results, additional research will be required to determine the full extent of aerobic exercise's clinical significance with regard to anxiety sensitivity. Several methodological considerations have been identified and should be improved upon in future investigations. These include the lack of a systematic evaluation of participants' previous exercise experiences, self-rated perceptions of effort while exercising, and exercise involvement between post-treatment and follow-up evaluations. Following is a brief discussion of each of these variables and the implications that they may have on the interpretation of the present results. 
In order to take part in the present study, participants were required to not be already involved in an aerobic exercise program. However, an in depth analysis of lifetime exercise history was not obtained. It is possible that people with diverse exercise experiences may respond very differently to vigorous exercise. Specifically, individuals who have participated in aerobic exercise in the past, particularly at higher intensities, may respond in different ways to exerciseinduced physiological arousal than someone who has never exercised. Consequently, exercise history represents a potential moderator variable that future research will need to evaluate to determine what, if any, impact it may have on the effects of aerobic exercise on anxiety sensitivity.

Although the discrepancy in average heart rates for the two treatment groups was found to be highly significant, perceptions of effort and physiological sensations experienced by group members were not directly assessed. Consequently, it is possible that individuals at the upper end of the low-intensity exercise group may have experienced many of the same sensations that were intended to be exclusive to the high-intensity exercise condition. Unfortunately, the potential impact that this overlap may have had on results remains unknown. However, it is unlikely that many of the participants in the low-intensity exercise condition achieved increases in physiological sensations similar to that of the high-intensity condition simply due to the discrepancy in speeds on the treadmill. Average treadmill speed for individuals in the highintensity exercise condition was approximately three times that of the comparison condition (2.91 vs. 1, respectively). Consequently, the overlap in body sensations between the two conditions was likely to be minimal. However, a more controlled evaluation of participants' perception of physical exertion is recommended.

A third potentially influential variable not assessed in the present study is possible changes in participants' exercise habits that may have occurred between post-treatment and follow-up. Although a relatively brief follow-up was employed in the present study, an increase in exercise intensity for even a few participants in the slow walking comparison condition could potentially have a relatively large impact on outcome measures at follow-up, particularly if high-intensity forms of exercise were undertaken. Future research may find it beneficial to systematically evaluate and control for changes in exercise habits following exercise treatment protocols in order to maximize the internal validity of the study and improve the confidence with which findings can be interpreted.

Generalizability of the present results is also somewhat limited by the selection criteria used. To have been selected for this study, individuals were required to endorse a relatively high level of anxiety sensitivity and be in good general health. They could not be involved in an aerobic exercise program, nor be receiving psychological or pharmacological treatment. Each of these requirements restricts the population to which these results are applicable. In a practical sense, however, clinicians would only use aerobic exercise as an intervention for individuals with high anxiety sensitivity if they were healthy and not already involved in an aerobic exercise program. However, research using a treatment-seeking population would strengthen the confidence with which the present results could be generalized. It should also be noted that one-third of the participants reported a history of panic attacks. Although this is at the upper bounds of normal 
limits for panic history in the general population (Rachman \& de Silva, 1996), it is consistent with the proposed relation between anxiety sensitivity and panic.

Clearly, there are limitations to using high-intensity aerobic exercise as an intervention for anxiety sensitivity. Individuals with certain health-related problems, such as heart disease or severe asthma, would not be candidates for this intervention. Also, some individuals may find it difficult to initiate or maintain a regular aerobic workout routine, and some may simply refuse to consider this option. However, for individuals with high anxiety sensitivity who cannot or will not consider more traditional means of intervention (i.e. pharmacology, psychotherapy) due to financial or personal constraints, aerobic exercise may prove to be an invaluable treatment alternative. Future research will also need to address whether longer participation in a highintensity exercise program (eight weeks or more) will confer additional benefits, and whether these benefits are maintained for longer follow-up periods.

\section{ACKNOWLEDGEMENTS}

This research is based in part on the master's thesis of the first author, supervised by the second author.

\section{REFERENCES}

Abadie, 1988 B.R Abadie, Relating trait anxiety to perceived physical fitness. Perceptual and Motor Skills, 67 (1988), pp. 539-543.

American College of Sports Medicine (ACSM), 2000 American College of Sports Medicine (6th ed.), ACSM's guidelines for exercise testing and prescription, Lippincott Williams and Wilkins, Baltimore (2000).

Chambless, Caputo, Bright and Gallagher, 1984 D.L Chambless, G.C Caputo, P Bright and R Gallagher, Assessment of fear of fear in agoraphobics: the Body Sensations Questionnaire and the Agoraphobic Cognitions Questionnaire. Journal of Consulting and Clinical Psychology, 52 (1984), pp. 1090-1097.

Clark, 1986 D.M Clark, A cognitive approach to panic. Behaviour Research and Therapy, 24 (1986), pp. 461-470.

de Coverley Veale, 1987 D.M de Coverley Veale, Exercise and mental health. Acta Psychiatricia Scandinavia, 76 (1987), pp. 113-120.

Ehlers, 1995 A Ehlers, A one-year prospective study of panic attacks: clinical course and factors associated with maintenance. Journal of Abnormal Psychology, 104 (1995), pp. 164-172. 
Fremont and Craighead, 1987 J Fremont and L.W Craighead, Aerobic exercise and cognitive therapy in the treatment of dysphoric moods. Cognitive Therapy and Research, 11 (1987), pp. 241-251.

Jacobson and Truax, 1991 N.S Jacobson and P Truax, Clinical significance: a statistical approach to defining meaningful change in psychotherapy research. Journal of Consulting and Clinical Psychology, 591 (1991), pp. 12-19.

Maller and Reiss, 1992 R.G Maller and S Reiss, Anxiety sensitivity in 1984 and panic attacks in 1987. Journal of Anxiety Disorders, 6 (1992), pp. 241-247.

McAuley, Mihalko and Bane, 1996 E McAuley, S.L Mihalko and S.M Bane, Acute exercise and anxiety reduction: does the environment matter?. Journal of Sport and Exercise Psychology, 18 (1996), pp. 408-419.

McEntee and Haglin, 1999 D.J McEntee and R.P Haglin, Cognitive group therapy and aerobic exercise in the treatment of anxiety. Journal of College Student Psychotherapy, 13 (1999), pp. 37-55.

McNally and Lorenz, 1987 R.J McNally and M Lorenz, Anxiety sensitivity in agoraphobics. Journal of Behavior Therapy and Experimental Psychiatry, 18 (1987), pp. 3-11.

McWilliams and Asmundson, 2001 L.A McWilliams and G.J.G Asmundson, Is there a negative association between anxiety sensitivity and arousal-increasing substances and activities?. Journal of Anxiety Disorders, 15 (2001), pp. 161-170.

Peterson and Reiss, 1992 R.A Peterson and S Reiss, Anxiety Sensitivity Index Revised Manual, International Diagnostic Systems Publishing Corporation, Worthington, OH (1992).

Rachman and de Silva, 1996 S Rachman and P de Silva, Panic disorder: The facts, Oxford University Press, Oxford (1996).

Reiss and McNally, 1985 S Reiss and R.J McNally, The expectancy model of fear, S Reiss, R.R Bootzin, Editors, Theoretical issues in behavior therapy, Academic Press, London (1985).

Reiss, Peterson, Gursky and McNally, 1986 S Reiss, R.A Peterson, D.M Gursky and R.J McNally, Anxiety sensitivity, anxiety frequency, and the prediction of fearfulness. Behaviour Research and Therapy, 24 (1986), pp. 1-8.

Roth, 1989 D.L Roth, Acute emotional and psychophysiological effects of aerobic exercise. Psychophysiology, 26 (1989), pp. 593-602.

Salmon, $2001 \mathrm{P}$ Salmon, Effects of physical exercise on anxiety, depression, and sensitivity to stress: a unifying theory. Clinical Psychology Review, 21 (2001), pp. 33-61.

Sexton, Maere and Dahl, 1989 H Sexton, A Maere and N.H Dahl, Exercise intensity and reduction in neurotic symptoms-a controlled follow-up study. Acta Psychiatricia Scandinavia, 80 (1989), pp. 231-235. 
Shephard, Cox and Simper, 1981 R.J Shephard, M.H Cox and K Simper, An analysis of "PARQ" responses in an office population. Canadian Journal of Public Health, 72 (1981), pp. 37-40.

Spielberger, Gorsuch, Lushene and Jacobs, 1983 C.D Spielberger, R.L Gorsuch, R.E Lushene and G.A Jacobs, State-Trait Anxiety Inventory for Adults, Consulting Psychologists Press, Palo Alto, CA (1983).

Taylor, 1999 S Taylor, Anxiety Sensitivity: Theory Research, and Treatment of the Fear of Anxiety, Lawrence Erlbaum Associates, New Jersey (1999).

Telch, Lucas, Schmidt, Hanna, Jaimez and Lucas, 1993 M.J Telch, J.A Lucas, N.B Schmidt, H.H Hanna, T.L Jaimez and R.A Lucas, Group cognitive-behavioral treatment of panic disorder. Behavior Research and Therapy, 31 (1993), pp. 279-287.

Zinbarg, Barlow and Brown, 1997 R.E Zinbarg, D.H Barlow and T.A Brown, Hierarchical structure and general factor saturation of the Anxiety Sensitivity Index: evidence and implications. Psychological Assessment, 9 (1997), pp. 277-284. 